Seraina Plotke

\title{
Hydrographie als poetisches Prinzip der Digression
}

\section{Die Flussbeschreibung Rhenus et eius descriptio elegans (1570) von Bernardus Mollerus}

Werke, die geographische oder topographische Phänomene beschreiben, hatten im 16. Jahrhundert Hochkonjunktur. Landstriche, Städte oder Gewässer, sie alle hat der frühe Buchdruck diskursiv vermessen, häufig verbunden mit der Schilderung der Sitten und Gebräuche der An- resp. Bewohner und unter Einbezug der neuen Möglichkeiten, die die Errungenschaften des Bild- und des Letterndrucks mit sich brachten: Verbale Beschreibungen wurden kombiniert mit oder ergänzt durch Holzschnitte oder Metallstiche, die Stadtansichten, Karten und anderes Illustrationsmaterial boten. Bereits in der Zeit der Inkunabeln findet man erste derartige Publikationen im Druck verwirklicht. Als bedeutendstes editorisches Großprojekt vor 1500 ist sicherlich die Schedelsche Weltchronik zu nennen, die mit ihrer historisch-temporalen Ausrichtung allerdings noch ganz in der Tradition mittelalterlicher Chroniken steht, indem sie heilsgeschichtlich argumentiert und das Strukturprinzip der Weltalter wählt. ${ }^{1}$ Nichtsdestotrotz spielen die Beschreibung räumlicher Gegebenheiten und topographischer Erscheinungen sowie die visualisierende Vermittlung und kartographische Illustration derselben in diesem frühen Druckerzeugnis eine richtungsweisende Rolle. ${ }^{2}$

Im Lauf des 16. Jahrhunderts treten Werke in den Vordergrund, die grundsätzlich spatial organisiert sind und den Fokus auf das Nebeneinander der darzustellenden Phänomene legen, indem sie die Welt nicht mehr zeitlich, sondern räumlich zu erfassen suchen, auch wenn historischen Dimensionen und chronographischen Erklärungsmustern nach wie vor große Bedeutung zukommt. Man

\footnotetext{
1 Siehe einführend und mit einer Übersicht zu den Ausgaben: Hartmann Schedel: Weltchronik. Kolorierte Gesamtausgabe von 1493. Faksimile-Nachdruck. Einleitung und Kommentar von Stephan Füssel. Augsburg 2004; Christoph Reske: Die Produktion der Schedelschen Weltchronik in Nürnberg / The production of Schedel's Nuremberg Chronicle. Wiesbaden 2000 (Mainzer Studien zur Buchwissenschaft 10). Weiterführend auch: Stephan Füssel (Hg.): 500 Jahre Schedelsche Weltchronik. Akten des interdisziplinären Symposions vom 23./24. April 1993 in Nürnberg. Nürnberg 1994 (Pirckheimer-Jahrbuch 9).

2 Das Verhältnis von chronographischer und topographischer Welterfassung in Schedels Werk erörtert Gernot Michael Müller: Die Germania generalis des Conrad Celtis. Studien mit Edition, Übersetzung und Kommentar. Tübingen 2001 (Frühe Neuzeit 67), S. 286-302.
} 
könnte von einer Art Paradigmenwechsel sprechen, der nicht zuletzt durch die Entdeckungsfahrten und die daraus gewonnenen Erkenntnisse geprägt war, sich aber auch aus der konsequenten Umsetzung der neuen drucktechnischen Möglichkeiten speiste. Paradebeispiel für ein Werk, das die Welt nach spatialen Maßgaben einzuholen sucht, und publizistische Meisterleistung zugleich ist Sebastian Münsters 1544 in Basel edierte Cosmographia, ${ }^{3}$ die sich ihrem Gegenstand sowohl verbal-beschreibend als auch illustrativ-dokumentierend nähert. ${ }^{4}$ Der Druck war dermaßen erfolgreich, dass er im Lauf der folgenden Jahrzehnte zahllose Neuausgaben, Erweiterungen und Übersetzungen erfahren hat. ${ }^{5}$

Die Cosmographia Münsters mag zwar das berühmteste und am aufwändigsten gestaltete Werk sein, das sich programmatisch räumlichen Erscheinungen widmet und diese für vielseitige weiterführende Erörterungen nutzt. Daneben kursierten im 16. Jahrhundert unzählige weniger bekannte Publikationen, die sich der Beschreibung spatialer Phänomene widmen: Literarische Stadtbeschreibungen, Deskriptionen topographischer Gegebenheiten, in Prosa oder in Versen gehalten, Schilderungen einzelner Erdteile oder politisch zusammenhängender Landstriche, lateinisch oder volkssprachlich, sie alle finden sich in großer Zahl, wenn auch nicht immer mit Illustrationen ausgestattet.

Im Kontext des Basler Buchdrucks, aus dem in der Jahrhundertmitte Münsters Cosmographia hervorging, ist die 1514 veröffentlichte Helvetiae descriptio des Schweizer Humanisten Heinrich Glarean zu nennen, die im Verbund mit einem panegyrischen Gedicht auf Kaiser Maximilian erschienen ist und eine lateinische Beschreibung und Lobpreisung der Schweiz und der einzelnen Kantone in Hexametern bietet. ${ }^{6}$ Die Dichtung besteht im Wesentlichen aus drei Teilen: Als

3 Der vollständige Titel der in Basel 1544 bei Heinrich Petri erschienenen Erstausgabe lautet: Cosmographia: Beschreibung aller Lender durch Sebastianum Munsterum in welcher begriffen, Aller völker Herrschafften, Stetten, und namhafftiger flecken, herkommen: Sitten, gebreüch, ordnung, glouben, secten, und hantierung, durch die gantze welt, und fürnemlich Teütscher nation: Was auch besunders in iedem landt gefunden unnd darin beschehen sey: Alles mit figuren und schönen landt taflen erklert, und für augen gestelt.

4 Mit Überblickscharakter zu Münsters Cosmographia siehe Matthew McLean: The Cosmographia of Sebastian Münster. Describing the world in the Reformation. Aldershot 2007 (St. Andrews studies in Reformation history); Günther Wessel: Von einem, der daheim blieb, die Welt zu entdecken. Die Cosmographia des Sebastian Münster oder Wie man sich vor 500 Jahren die Welt vorstellte. Darmstadt 2004.

5 Vgl. Karl Heinz Burmeister: Sebastian Münster. Eine Bibliographie. Wiesbaden 1964.

6 Das Titelblatt des 1514 bei Adam Petri in Basel veröffentlichten Drucks kündigt die Werke wie folgt an: Ad divum Max. Aemilianum Romanorum imperatorem semper Augustum, Henrici Glareani Helvetii poe. laure. panegyricon; Eiusdem de situ Helvetiae \& vicinis gentibus: de quattuor Helvetiorum pagis: pro iustissimo Helvetiorum foedere panegyricon. Eine Übersetzung von Glare- 
Erstes werden die Landesgrenzen nachvollzogen, die wichtigsten Flüsse genannt und die topographische Beschaffenheit der Schweiz geschildert, wobei Glarean mit der Feststellung schließt, nicht einmal Vergil oder Homer hätten die Schönheit der betreffenden Gewässer gebührend preisen können. Unmittelbar daran knüpft der zweite Teil des Werks an, die Skizzierung der vier Regionen der Schweiz. Glarean spricht in diesem Zusammenhang von pagus und versteht darunter jeweils dasjenige Gebiet, das den Lauf eines Flusses begleitet: So gesehen würden die vier Flüsse Thur, Limmat, Reuss und Aare die Regionen der Schweiz bestimmen. Der Zwischentitel Panegyricum leitet den letzten Teil der Dichtung ein, der sich den zwölf Orten der damaligen Eidgenossenschaft widmet. Nacheinander werden Zürich, Bern, Luzern, Uri, Schwyz, Unterwalden, Zug, Glarus, Basel, Freiburg, Solothurn, Schaffhausen und, als Nachtrag, Appenzell besungen - Letztes war 1513 gerade erst als dreizehnter Ort in den Bund der Eidgenossen aufgenommen worden. Der Humanist hebt das Besondere jedes einzelnen Kantons hervor, indem er beispielsweise das Berner Münster mit den Pyramiden von Memphis vergleicht oder die Männer von Zug mit dem Römer Camillus. Glareans Werk erreichte eine interessierte Leserschaft, was zur Folge hatte, dass fünf Jahre später eine kommentierte Ausgabe mit ausführlichen Prosaanmerkungen von Oswald Myconius erschien. ${ }^{7}$

In die Fußstapfen Glareans trat sein Schüler Aegidius Tschudi - um ein weiteres Basler Druckbeispiel zu nennen -, der 1538 seine in deutscher Sprache gehaltene Beschreibung der Schweizer Gebirgswelt veröffentlichte. ${ }^{8}$ Nicht zuletzt

ans Werk bietet die Ausgabe: Henricus Glareanus: Helvetiae descriptio - Panegyricum. Hg. und übers. von Werner Näf. St. Gallen 1948.

7 Das kommentierte Werk erschien 1519 ebenfalls in Basel, nun aber in der Offizin von Johannes Froben. Im Anschluss an die Beschreibung der Schweiz wird noch einmal das Panegyricon auf Kaiser Maximilian abgedruckt, wie auch der Wortlaut des Titelblatts dieser Ausgabe verdeutlicht: Descriptio de situ Helvetiae, \& vicinis gentibus / per eruditissimum virum Henricum Glareanum Helvetium, poëtam laureatum; Idem de quatuor Helvetiorum pagis; Eiusdem pro iustissimo Helvetiorum foedere Panegyricon / cum commentariis Osvaldi Myconii Lucernani; Ad Maximilianum Augustum Henrici Glareani Panegyricon. Siehe weiterführend zu Glareans Werk und Myconius’ Kommentar: Jean-Daniel Moreod, Anton Näf: Guillaume Tell et la libération des Suisses. Lausanne 2010, S. 81-87.

8 Der vollständige Titel des bei Johannes Bebel 1538 in Basel edierten Werks lautet: Die uralt warhafftig Alpisch Rhetia sampt dem Tract der anderen Alpgebirgen : nach Plinij Ptolemei Strabonis auch anderen Welt und gschichtscheybern [sic] warer anzeygung / durch den Ehrnvesten und wysen herren herr Gilg Tschudi von Glarüs ettwo in Sarganser land darnach zuo Baden im Ergöw gmeiner Eydgnossen Landvogt in Tütsch spraach zuosamen getragen und yetz mit einer Geographischen tabel ussgangen. Wichtige Beiträge zu Aegidius Tschudi vereint der Band: Katharina Koller-Weiss, Christian Sieber (Hgg.): Aegidius Tschudi und seine Zeit. Basel 2002. 
um das Werk der gesamten humanistischen Bildungswelt Europas zugänglich zu machen, wurde noch im selben Jahr eine von Sebastian Münster angefertigte lateinische Übersetzung der Abhandlung unter dem Titel De prisca ac vera Alpina Rhaetia [...] descriptio herausgegeben. Als Beigabe, bereits zur volkssprachlichen Publikation, erschien die gesüdete Karte Helvetiae descriptio, die erste relativ genaue Karte der Schweiz. ${ }^{9}$

Auf Aegidius Tschudis Beschreibung der Schweiz wiederum basiert jenes Werk - zumindest in dessen ersten Teil -, das im Zentrum der folgenden Überlegungen zur Hydrographie stehen soll und das, so die These, die dichterische Form der Flussbeschreibung zu einem eigenen poetischen Prinzip der Digression erhebt. Nicht nur mit der Schweiz und ihren Flüssen und Bergen, sondern mit dem größten mitteleuropäischen Strom in seiner gesamten Länge beschäftigt sich das umfangreiche lateinische Lehrgedicht Rhenus et eius descriptio elegans des Münsteraner Kanonikers Bernardus Mollerus, das erstmals 1570 in Köln publiziert und 1596 mit modifiziertem Titel zusammen mit einer ausfaltbaren Karte des Rheins und seiner Zuflüsse aufgelegt wurde. ${ }^{10}$ Die Dichtung ist in sechs Bücher von je 1000 bis 1500 Versen eingeteilt, beschrieben wird der Rhein von der Quelle bis zur Mündung, wobei sich die Schilderung durch unzählige inhaltliche Abschweifungen - kleine Geschichten, Aitien, mythische Erzählungen - auszeichnet, die die Darstellung bereichern. Dediziert ist das in elegischen Distichen gehaltene Werk Johann Graf von Hoya, Fürstbischof der Diözesen Münster, Osnabrück und Paderborn, wie das ausführliche, ebenfalls in Distichen formulierte Proömium eröffnet. Jedem Buch ist eine kurze Prosazusammenfassung vorgeschaltet, die skelettartig die Ortschaften und Zuflüsse aufführt, welche den jeweils beschriebenen Flussabschnitt prägen. ${ }^{11}$

9 Die nur in wenigen Exemplaren erhaltene Karte ist als Nachdruck erschienen: Aegidius Tschudi: Nova Rhaetiae descriptio atque totius Helvetiae 1538. Erste Karte der Schweiz. Zürich 1962.

10 Der vollständige Titel des in Köln bei Johannes Birckmann veröffentlichten Erstdrucks lautet: Rhenus et eius descriptio elegans a primis fontibus usque ad Oceanum Germanicum ubi Urbes, Castra, \& Pagi adiacentes, Item flumina \& rivuli in hunc influentes, \& si quid praeterea memorabile occurat plenissime carmine Elegiaco depingitur. Nach der Erstausgabe 1570 erfolgte 1571 eine weitere Auflage. 1596 wurde das Werk erneut ediert, nun bei Peter Haack in Köln, unter dem Titel: D. Bernardi Molleri Monasteriensis Rheni A Primis Fontibus Usque Ad Oceanum Germanicum Descriptio: In Qua non solum Urbes, Castra, \& Pagi adiacentes; item Flumina \& rivuli in hunc influentes, depinguntur: verum etiam plurimae Antiquitates, variae historiae, \& multa scitu digna [...]. 11 Die Aufteilung des Rheins auf die sechs Bücher stimmt nicht mit der heutigen Unterteilung in Alpenrhein, Hochrhein, Oberrhein etc. überein, Mollerus hat sich im Wesentlichen an den wichtigeren Zuflüssen orientiert (mit Ausnahme des Mains): Im ersten Buch beschreibt er den Rhein von den beiden Quellen bis zum Zusammenfluss mit der Aare, das zweite Buch geht von Koblenz an der Aare-Mündung bis Mannheim (also bis zum Neckar-Zufluss), das dritte von Mannheim 
Bemerkenswert und signifikant ist bei Mollerus' Dichtung bereits der Umstand, dass die Überschrift im Erstdruck nicht etwa Rheni descriptio lautet, wie naheläge und man im Gefolge der oben erwähnten Werke erwarten würde, sondern aus der Zweigliedrigkeit des Haupttitels Rhenus et eius descriptio elegans rezeptionssteuernde Sinnschichten erwachsen. Indem das Augenmerk zunächst auf den Rhein als solchen gelenkt wird und erst im zweiten Schritt die Perspektivierung auf die descriptio erfolgt, knüpft das Werk einerseits an bekannte Flussgedichte wie die Mosella des Ausonius aus der Spätantike und an dessen humanistische Nachfolger an, in deren Kontext es damit gestellt wird. ${ }^{12}$ Andererseits wird durch die doppelte Pointierung verdeutlicht, was den Leser erwartet, nämlich ein Gedicht, das den Namen Rhenus trägt und - quasi darüber hinaus eine Beschreibung des betreffenden deutsch-europäischen Stromes bietet. Zugespitzt formuliert heißt dies: Das präsentierte Gedicht wird mit dem Fluss, als dessen Abbild es über das erste Titelwort annonciert wird, gleichgesetzt.

Dass die vielen tausend Verse nicht nur eine Beschreibung des Stromes von den Quellen bis zur Mündung liefern, wie der Untertitel hervorhebt - a primis fontibus usque ad Oceanum Germanicum -, sondern gleichsam selbst zum Abbild und Sinnbild des Flusses erhoben werden, veranschaulicht nicht nur die außergewöhnliche Länge des Rheingedichts, die alle früheren Flussbeschreibungen um ein Vielfaches übersteigt und damit die besondere Erstreckung dieses Fließgewässers spiegelt. Auch im Proömium wird die Vorstellung der Dichtung im Fluss - oder auch: des Dichtwerks als Fluss - mehrfach reflektiert. Bereits der

bis zur Mosel-Mündung, das vierte von der Mosel bis nach Köln (nördlich der Sieg-Mündung), das fünfte Buch reicht von Köln bis zum Zusammenfluss von Rhein und Lippe und das sechste schließlich von dort bis zur Nordsee. Eine detailliertere Übersicht über den gesamten Aufbau des Werks sowie einführende Informationen zum Autor bietet dankenswerterweise der Beitrag von Beate Hintzen: Ein Münsteraner in einer rheinischen Metropole: Bernardus Mollerus' Blick auf Köln (S. 69-81 im vorliegenden Band). Die dort (S.69) vertretene Auffassung von Mollerus als poeta minimus teile ich allerdings aus noch auszuführenden Gründen nicht (abgesehen davon, dass ich diese Kategorie grundsätzlich für problematisch halte). Zur Einordnung des Werks in verschiedene Gattungstraditionen siehe die beiden früheren Aufsätze der Autorin: Beate Czapla: Der Rhein, Europas Strom, nicht Deutschlands Grenze. Bernardus Mollerus' Rhenus et eius descriptio elegans und die Tradition lateinischer Flussdichtung in Europa. In: Jahrbuch für Internationale Germanistik 30 (1998) 2, S. 8-31; Beate Czapla: Neulateinische Lehrdichtung zwischen der literarischen Tradition von Hesiod bis Manilius und der neuzeitlichen Ars apodemica am Beispiel von Bernardus Mollerus' Rhenus und Cyriacus Lentulus' Europa. In: Neulateinisches Jahrbuch 1 (1999), S. 21-48. Ebenfalls Bernardus Mollerus und seiner Rheindichtung gewidmet ist der Beitrag von Thomas Gärtner: Die frühen Werke des Münsteraners Bernhardus Mollerus: der Rhenus und die Ecclesias (S. 94-105 im vorliegenden Band).

12 Siehe dazu eingehend Czapla: Der Rhein (Anm.11). 
Auftaktvers rekurriert auf die Fluktualität und Vergänglichkeit von Flusswasser: Scribere decrevi, quid scribam praeterit (V.1). ${ }^{13}$ Mollerus kündigt an, über etwas schreiben zu wollen, das während des Schreibprozesses gerade vorübergeht und verrinnt, und macht mit diesem Eingang explizit auf die entscheidende Qualität des Dahinströmens von Flüssen aufmerksam. Flusswasser besitzt die Eigenschaft der scheinbaren Substanz: Es ist für den Betrachter zwar da, jedoch nicht mit sich selbst identisch. Flusswasser lässt sich nicht im Moment festhalten, sondern ist an einer bestimmten Stelle im Prozess der Flüchtigkeit immer schon weiter- und vorbeigeflossen.

Von diesem Gedanken her lassen sich Flüsse als solche sowie Flusswasser in sinnbildliche Analogiebeziehungen zu Dichtwerken und deren Versfolgen setzen, ${ }^{14}$ wobei die Dimensionen von Zeit und Raum bei Flüssen in einem ähnlich paradoxen Verhältnis stehen wie bei schriftlich festgehaltenen Versgedichten. Einerseits ist die topographische Ausdehnung des Flusslaufs im Gelände räumlich zu denken, dem entsprechend lässt sie sich schließlich auch kartographisch festhalten. Analog dazu besitzt das literarische Produkt eine Erstreckung von vielen aufeinanderfolgenden Zeilen - etwa von mehreren tausend Versen, wie im Fall des Rhenus -, die skriptural auf den Buchseiten eine räumliche Ausdehnung besitzen. Wie die Spur auf der Karte, die den Flusslauf in der Fläche abbildet, konstituiert auch die Buchstabenfolge der Dichtung eine Linie, die in der Handschrift oder im Druck zunächst einmal eine spatiale Dimension aufweist.

Auf der anderen Seite lässt sich das Gedicht nur in der Zeitlichkeit und durch Verzeitlichung rezipieren: Die Wörter und Verse werden ausgesprochen oder still gelesen, wobei sie sich mit dem Fortschreiten der Lektüre sofort wieder verflüchtigen, ähnlich wie der Verlauf des Flusses von der Quelle bis zur Mündung nur temporal verfolgt werden kann und die jeweiligen Positionen nach dem Passieren

13 Die Versangaben zum Proömium richten sich nach der Ausgabe Mollerus: Rhenus (Anm.10). Mit dem Proömium des Werks setzt sich auch Gärtner (Anm. 11) auseinander, jedoch mit abweichenden Einschätzungen. Den Auftaktvers versteht Gärtner anders (vgl. ebd., S. 95-99).

$14 \mathrm{Zu}$ solchen und ähnlichen Überlegungen siehe insbesondere: Alexander Honold: Der Rhein. Poetik des Stroms zwischen Elementarisierung und Domestikation. In: Anglia. Zeitschrift für Englische Philologie 126 (2008) 2, S. 330-344. Des Weiteren auch: Alexander Honold: Vom Rhein zur Donau und zurück. Die Bedeutung der deutschen Ströme in der Wiederentdeckung und Mythisierung des Nibelungenstoffs. In: Schätze der Erinnerung. Geschichte, Mythos und Literatur in der Überlieferung des Nibelungenliedes. Dokumentation des 7. Wissenschaftlichen Symposiums der Nibelungenliedgesellschaft Worms e. V. und der Stadt Worms vom 17. bis 19. Oktober 2008. Hg. von Volker Gallé. Worms 2009 (Schriftenreihe der Nibelungenliedgesellschaft Worms 6), S.117-145; Alexander Honold: Metamorphosen. Ovid und die Mythopoetik des Wassers. In: Mythopoetik in Film und Literatur. Hg. von Matthias Bauer, Maren Jäger. München 2011 (Projektionen 5), S. 33-46. 
der Stellen unverweilt aus dem Blickfeld verschwinden und der Vergangenheit angehören. Die Lektüre eines Dichtwerks gleicht dem Entlangfahren an einem Fluss. Wie in der Nennung der einzelnen Ortschaften und der beschreibenden Abfolge der Zuflüsse des Rheins stromabwärts deutlich wird, realisiert Mollerus' Rhenus dieses raum-zeitliche Abbildungsverhältnis mitunter punktgenau.

Schon das Proömium beutet die abbildlichen und sinnbildlichen Beziehungsgeflechte von Fließgewässern und Dichtwerken poetologisch aber noch weiter aus. So wünscht sich der Dichter in dessen Mitte emphatisch: In mea se fundat dominator carmina Rhenus (V.134). Zum sinnbildlich nutzbaren Bedeutungsreservoir von Flüssen gehört die Vorstellung der Wasserkraft, die sich mit Gewalt die Bahnen sucht, in denen das Gewässer seinen Lauf nimmt. ${ }^{15}$ Der Rhein möge zum Lenker und Meister seiner Verse werden, sie mit seinem Quell bespülen, fordert Mollerus an dieser Stelle. Das Flusswasser des Rheins spendet dem Dichtwerk Kraft, überträgt damit auch kompositorische Energie.

Dass die Stärke und Wucht des Wassers allerdings positiv oder negativ konnotiert sein kann, wird sogleich reflektiert, wenn es im darauffolgenden Pentameter heißt: In mea se Rhenus praebeat ora levem (V.135). Mit behänder Leichtigkeit zeige sich der Fluss auf der Zunge des Dichters - die Vorstellung des den Mund erquickenden Brunnquells schwingt hier unweigerlich mit. Das Wasser als Leben und Verderben bringende Substanz ist Mollerus in seiner Ambivalenz stets präsent. Schon die im Titel gewählte Auszeichnung der descriptio als elegans bringt die Widersprüchlichkeit, die in der Komposition des gedichteten Fließgewässers steckt, auf den Punkt: Zur Naturgewalt des Wassers gehört, dass sie sich schwer einzwängen lässt, der Fluss als elegische Form also immer schon ein Kulturprodukt sein muss. ${ }^{16}$

In diesem Spannungsverhältnis bewegt sich die Ankündigung, die das Proömium einige Verse später formuliert, indem der Dichter eindringlich festhält: RHENUS erit carmen: Rheni narrare fluenta / Destino: materies competit ista mihi (V.160 f.). Mit diesem Distichon setzt Mollerus den Rhein und sein Gedicht ausdrücklich in eins, lässt sie beide identisch werden. Er bestimmt das Flusswasser als seinen Stoff, pointiert den Entschluss, den Rhein in seinem Fließen zu erzählen. Programmatisch wird die Kongruenz der strömenden Naturgewalt mit dem dichterischen Produkt bekannt gegeben: Das Gedicht und das Gewässer, sie fordern gemäß Mollerus in ähnlicher Weise ihr Recht.

Dass Flusswasser - und zwar dasjenige eines so monumentalen Stroms wie des Rheins - Pate für Mollerus' poetisches Konzept steht, wird in den sechs

15 Dazu Honold: Der Rhein (Anm. 14), S. 337.

16 Vgl. ebd., S. 332. 
Büchern des Lehrgedichts selbst augenscheinlich. Dies sei exemplarisch anhand des ersten Buchs demonstriert, das den Flusslauf von der doppelten Quelle des Vorder- und des Hinterrheins bis zur Einmündung der Aare im Norden der Schweiz verfolgt und insofern an die oben genannten Basler Drucke anschließt, als Mollerus, der selbst nie in dieser Gegend war, als Informationsquelle für die geographischen Details offensichtlich Aegidius Tschudis Beschreibung der Schweiz benutzte. Was sich am Beispiel des ersten Buchs an poetischen Techniken und hydrographischen Verfahrensmustern dokumentieren lässt, findet sich jedoch auch in sämtlichen anderen Büchern des Rhenus und ist charakteristisch für Mollerus' dichterische Vorgehensweise.

In seiner Grobstruktur berichtet das erste Buch der Reihe nach von den Ortschaften und Zuflüssen, die den Alpenrhein und den Hochrhein bis zur AareMündung prägen. Wie im Vorüberziehen werden die beiden im Gebirge entspringenden Quellflüsse und der sich nach der Vereinigung von Vorder- und Hinterrhein fortbildende Wasserlauf beschrieben, werden die wichtigsten Siedlungen und Städte direkt am Rhein sowie in dessen näherer Umgebung genannt: Chur, Maienfeld, Sargans, Bludenz, Feldkirch, Bregenz, Lindau, Konstanz, Steckborn, Diessenhofen, Schaffhausen, Zurzach und Eglisau. Eingepasst in diese Folge erwähnt der Text geographische Besonderheiten im weitesten Sinn, etwa die Bodenseeinseln Mainau und Reichenau oder natürlich auch den Rheinfall. Einen speziellen Status besitzen die größeren Zuflüsse zum Rhein. Die betreffenden Mündungen geben Anlass, diese Flüsse selbst wiederum exkursartig in ihrem Lauf einzuholen, was dazu führt, dass das letzte Drittel des ersten Buchs gar nicht dem Rhein, sondern Reuss, Limmat und Aare gewidmet ist.

Zum besonderen poetischen Prinzip von Mollerus gehört, dass die skizzierte Grobstruktur der Flussläufe - sowohl des Rheins als auch seiner Zuflüsse - den Text des Gedichts immer wieder zu kleineren oder umfangreicheren Digressionen leitet. Wie sich Wasser und insbesondere Flusswasser seine Wege in Verästelungen sucht, Bahnen abzweigt oder sich gabelt - vor allem vor den großen Gewässerkorrektionen des 19. Jahrhunderts ${ }^{17}$-, so führt das Gedicht ein Eigenleben

17 Wie sich Flüsse in ihrem natürlichen Gefälle verhalten, ist uns in Mitteleuropa kaum mehr bewusst, da die großen Gewässerkorrektionen und Entsumpfungsmaßnahmen der vergangenen zwei Jahrhunderte die Ströme heute völlig anders aussehen lassen als früher. Rekonstruktionen und alte Karten zeigen, wie verästelt der ursprüngliche Lauf des Rheins über weite Strecken war, der sich immer wieder in mehrere Parallel- und Nebenarme aufspaltete, in Vergabelungen bisweilen ganz versiegte, öfters auch starke Mäander aufwies, wo er heute in einem geraden Betonbett, unterbrochen von Wasserkraftwerken und Schleusen, kanalisiert ist. Siehe dazu weiterführend: David Blackbourn: The Conquest of Nature. Water, Landscape and the Making of Modern Germany. New York, London 2006, dt.: Die Eroberung der Natur. Eine Geschichte der deutschen 
dadurch, dass von der eigentlichen Beschreibung des Flusslaufs abgeschweift wird, Überlegungen und Geschichten eingeschoben werden, die von der Hauptthematik des Rheins wegführen, sich mit dem Strahl der Flussbeschreibung kreuzen und später zu ihr zurückfinden oder einfach in einer Nebenlinie versanden.

Die derart eingefügten Schilderungen und Gedankengänge präsentieren ganz unterschiedliche Themen, können sich auf kurze Einsprengsel beschränken, aber auch einen Umfang von mehreren Dutzend Distichen aufweisen. Die ausgedehnteste Digression des ersten Buchs erstreckt sich über mehr als 200 Verse. Sie steht an signifikanter Stelle und nimmt ein für den Flusslauf bedeutsames Phänomen zum Anstoß der Abschweifung. Es geht um den schon bei Aegidius Tschudi als Scala mons bezeichneten Schollberg bei Sargans, ${ }^{18}$ vor dem der Rhein eine scharfe Biegung nach rechts zum Bodensee hin nimmt, wobei Mollerus das sich an die Kurve anschließende Binnendelta ebenfalls in seine digressiven Überlegungen einbaut.

Der Münsteraner nimmt die Erwähnung des mons Scala in seinem RheinGedicht zum Anlass einer ausführlichen Hommage an Paul Skalich und das Veroneser Fürstengeschlecht della Scala, wobei das sich zum Bodensee öffnende Flussdelta über das griechische Wort skalenós (schief, ungerade) - das insbesondere auch ein ungleichseitiges Dreieck bezeichnet - in die inhaltlichen Verästelungen mit eingebunden wird. ${ }^{19}$ Was Mollerus in diesem Exkurs unterbreitet, sind Herleitungen des Adelsgeschlechts der Scaliger und deren Repräsentanten, die wilde Etymologien beinhalten und historisch wie geographisch bis zum Nildelta des alten Ägypten ausgreifen. Diese Deduktionen selbst haben Flusscharakter, indem sie sich in der Argumentation verzweigen und kaum stringent $\mathrm{zu}$ begreifen sind, um zuletzt jedoch in die ebenfalls ausufernde Ehrung Paul Skalichs zu münden, ${ }^{20}$ die damit quasi das sedimentreiche Binnendelta des Bodensees repräsentiert.

Bernardus Mollerus hatte den Gelehrten Paulus Scalichius oder Scaliger, wie er sich auch nannte, im Umfeld des Münsteraner Fürstbischofs Johann von Hoya kennengelernt, wo sich dieser in den späten 1560er Jahren aufhielt. ${ }^{21}$ In Wahrheit stammte Skalich aus einfachen Verhältnissen und war in Zagreb geboren, er gab

Landschaft. Übers. von Udo Rennert. München 2006; Eberhard Henze: Technik und Humanität. Johann Gottfried Tulla. Mannheim 1989.

18 Vgl. Tschudi (Anm. 8), S. Jiiv .

19 Vgl. Mollerus: Rhenus (Anm. 10), S. 17-25.

20 Vgl. ebd., insbes. S. 21-25.

21 Vgl. auch Czapla: Der Rhein (Anm.11), S. 29. 
sich aber über Jahrzehnte als Abkömmling der Veroneser Adelsfamilie Scaliger aus. ${ }^{22}$ Für Mollerus war Skalich - wie für Johann von Hoya und für viele andere auch - kein Hochstapler, sondern der bekannte adlige Humanist, der sich durch Werke theologischen und philosophischen Inhalts hervorgetan hatte, insbesondere durch die Schrift Encyclopaediae, seu orbis disciplinarum tam sacrarum quam prophanarum epistemon, die 1559 in Basel bei Johannes Oporin erschienen war.

So war es wohl der schon im Titel dieses Werks genannte Gedanke des Enzyklopädischen, der Mollerus bewog, Skalich mittels der Digression eine solch prominente Stellung in seinem Rhein-Gedicht einzuräumen, ja diesen geradezu zum heimlichen Widmungsträger zu erheben. Denn nicht nur die Länge der Abschweifung, sondern auch ihre besondere Position innerhalb des Rheinlaufs markiert diese Verse als bemerkenswert: Der zum Anlass genommene Scala mons stellt eine Art Gegenkraft gegen das Wasser dar; der Berg ist es, der den Rhein zur Krümmung und Veränderung seines geraden Laufs zwingt. ${ }^{23}$ Mollerus pointiert mit seinem Exkurs im Grunde genommen die Festigkeit und Resistenz des Scala mons, die es braucht, um den Gewalten des Flusswassers zu trotzen - genau so, wie Kunstvermögen und enzyklopädische Bildung notwendig sind, um die Wildheit des Rhein-Gewässers in verspoetische Bahnen zu lenken.

Das Geschlecht der Scaliger und ihr (vermeintlicher) Abkömmling Paulus Scalichius werden von Mollerus also über den Scala mons und die mit diesem verbundene Abschweifung $\mathrm{zu}$ Begründern der dichterischen Potenz stilisiert,

22 Eine minutiöse Rekonstruktion von Skalichs Werdegang bietet Gerta Krabbel: Paul Skalich. Ein Lebensbild aus dem 16. Jahrhundert. Münster 1915 (Geschichtliche Darstellungen und Quellen 1). Der erste Teil dieser Abhandlung ist zudem erschienen als: Gerta Krabbel: Aus Paul Skalichs Leben. Münster 1914. Ebenfalls ausführlich mit Skalich beschäftigt sich: Ludwig von Baczko: Geschichte Preußens. Bd. 3. Königsberg 1794, S. 270-291. Auf der Basis dieser Untersuchungen lassen sich die Stationen von Skalichs Lebenslauf bis zu seinem Münsteraner Aufenthalt wie folgt nachzeichnen: Im Jahr 1534 geboren und als Kind durch den Laibacher Bischof Urban Textor gefördert, konnte Skalich in Wien und Bologna studieren und den Doctor Theologiae erwerben. Von Kaiser Ferdinand I. zum Hofkaplan ernannt, fiel er nach und nach durch Lügengeschichten auf, verließ Wien 1557 und durfte, da er in Ungnade gefallen war, nicht wieder dahin zurückkehren. Er hielt sich darauf in diversen deutschen Städten und bei verschiedenen Adelsgeschlechtern auf und nannte sich fortan Paul Scaliger, Fürst de la Scala, Landherr des römischen und Heergraf des ungarischen Reichs, Heergraf zu Hunn und Markgraf zu Verona. Ab 1561 befand er sich in Königsberg bei Herzog Albrecht von Preußen, wurde aber von dortigen Adligen bald als Schwindler bezeichnet. Er floh 1566 zunächst nach Paris und dann nach Münster, wo er sich wiederum beim Fürstbischof einzuschmeicheln vermochte.

23 Topologisch betrachtet ist es nicht der Schollberg, der den Rhein zur Biegung zwingt, sondern eine nur wenige Meter hohe Schwelle vor dem Walensee, die im Lauf der Jahrtausende zu einer Talwasserscheide führte (vgl. etwa Alexander Supan: Grundzüge der physischen Erdkunde. Paderborn 2013 [Ndr. der Ausg. von 1916], S. 704-710). 
zum Ausgangspunkt künstlerischer Kreativität erhoben. Wie oben bereits angedeutet, lässt sich das aufwändig in die Digression eingewobene Rheindelta des Bodensees darüber hinaus als Sinnbild für poetische Fertilität lesen: Gerade das Nildelta - und der Text rekurriert explizit auf den Nil - gilt schließlich als Inbegriff der Fruchtbarkeit. Auf Grund der Analogiebeziehung zum Flussdelta wird Paul Skalich gewissermaßen zum verästelten Nährboden der enzyklopädischen Poesie stilisiert.

Abschließend können folgende Beobachtungen zu Mollerus' Rhenus et eius descriptio elegans festgehalten werden. Nimmt man das spezifische - eingangs erläuterte - Raum-Zeit-Gefüge von Fließgewässern zum Ausgangs- und Vergleichspunkt, lässt sich die Dichtung als Umsetzung mehrerer sich überlagernder poetologischer Konzepte lesen, die nicht nur mit den im sechzehnten Jahrhundert durch den Buchdruck populär gewordenen Kulturtechniken der Kartographie und der enzyklopädischen Kosmographie zusammenhängen, sondern genuin mit dem Problem der Verknüpfung von Natur und Kultur als künstlerischer Leistung.

Aus der Raumperspektive betrachtet, und damit auch aus derjenigen der Kartographie, könnte man sagen, dass die Flussläufe sowohl des Rheins als auch seiner großen Zuflüsse für Mollerus die Matrix bilden, um eine detaillierte verspoetische Landkarte (hier im ersten Buch: der Schweiz) zu zeichnen, wobei das strukturbildende Prinzip der Dichtung als Fluss - und im Fluss - mitunter zu Gunsten der Vollständigkeit der Graphik aufgegeben wird, so etwa ganz zum Schluss des ersten Buchs, als mit Biel und Murten Städte erwähnt werden, die - zumindest damals, also vor der betreffenden Aarekorrektion - nicht in der Nähe der Aare lagen. Zur kartographischen Methode gehört, dass die im Zusammenhang mit dem Flusslauf genannten Städte zwar angeführt, in der Regel aber nicht näher beschrieben werden. Das poetische Werk konstituiert also quasi eine hydrographische Karte, in der die Städte und Ortschaften gleichsam als Punkte eingezeichnet sind. Diese Pointierung der Dichtung wird in der späteren Ausgabe von 1596 stark gemacht, die die ursprüngliche zweigliedrige Titelformulierung $\mathrm{zu}$ Rheni ... descriptio abändert ${ }^{24}$ und in den Druck eine große, ausfaltbare Karte Mitteleuropas einbindet. ${ }^{25}$

Parallel zu dieser spatialen Perspektivierung wird in Mollerus' Rhenus die Engführung von Wasserkraft und Dichtkunst in der fließenden Temporalität

24 Zum vollständigen Titel der Ausgabe von 1596 siehe Anm. 10.

25 Die Karte ist gewestet und zeigt ganz links die Schweizer Alpen, rechts die Nordsee, so dass für den Flusslauf des Rheins optimale Darstellbarkeit erreicht wird (vgl. die Reproduktion in diesem Band). 
anschaulich gemacht - nicht umsonst spricht man ja auch metaphorisch vom ,Fluss der Zeit‘. Der Dichter erhebt das Strömen von Fließgewässern zum poetischen Prinzip, indem er immer wieder abschweift und seine Beschreibung verästelt, wie dies die mäandrierenden und sich in Nebenarme verzweigenden Flüsse in ihrer natürlichen Gestalt tun. Das Sprudeln und Schleifen-Ziehen des Wassers zeigt sich im Rhenus denn bis in das dichterische Verfahren, mit Hilfe von Wortwiederaufnahmen, Wiederholungen und Umformulierungen das Nicht-Gradlinige auch in den einzelnen Versen zu spiegeln. ${ }^{26}$ Mollerus erhebt den Rhein als deutsch-mitteleuropäischen Strom damit zum Analogon des Dichtwerks. In einer Zeit, in der die Kartographie zu blühen begonnen hat und mittels der Technik des Kupferstichs Landkarten in allen Dimensionen angefertigt werden, komponiert der Münsteraner mit seinem Flussgedicht eine hydrographische Abbildung Mitteleuropas, wobei der Rhein das Grundgerüst bildet, um über Digressionen nicht nur in die weiteren Verästelungen des Geländes vorzudringen, sondern dem Poetisch-Enzyklopädischen seinen Lauf zu lassen.

26 Insofern interpretiere ich die vielen Wiederholungen und scheinbar schwerfälligen Formulierungen im Text nicht als Unvermögen des Dichters (vgl. Hintzen [Anm. 11], S. 69), sondern als bewusst gewähltes poetisches Prinzip, das in der Nachahmung des mäandrierenden Fließgewässers besteht. 